\title{
Ozonoterapia como adyuvante en el tratamiento periodontal no quirúrgico y quirúrgico.
}

\author{
Ozonoterapia as adjuvant in the non-surgical \\ and surgical periodontal treatment.
}

\begin{abstract}
Jorge Luis Salazar-Leal,* Rubén Gerardo Lozano-Belaunzarán,* Gloria Martínez-Sandoval,* María de los Ángeles Andrea Carvajal-Montes de Oca,* María Gabriela Chapa-Arizpe*
\end{abstract}

\section{RESUMEN}

Introducción: El ozono $\left(\mathrm{O}_{3}\right)$ presenta múltiples acciones biológicas, entre ellas su efecto antimicrobiano, lo que ha sido beneficioso en odontología, siendo la presentación acuosa la más utilizada ( $20 \mu \mathrm{g} /$ $\mathrm{mL}$ ), la cual presenta efectos similares a la clorhexidina. Reporte de caso: Paciente masculino de 76 años de edad, diagnosticado con periodontitis crónica moderada localizada, el cual fue tratado mediante ozonoterapia acuosa durante la fase inicial periodontal y la fase quirúrgica en colgajo por debridación. 12 semanas posteriores al acto quirúrgico se obtuvo la eliminación de las bolsas periodontales, encontrándose un surco de 3 y 2 mm y un buen control de placa dentobacteriana. Conclusión: No existen reportes acerca del uso de ozonoterapia acuosa durante un colgajo por debridación. El éxito del tratamiento periodontal consiste en la eliminación del factor causal así como en establecer y mantener un control de placa dentobacteriana adecuado.

Palabras clave: ozono, ozonoterapia, periodontitis.

\section{ABSTRACT}

Introduction: Ozone $\left(\mathrm{O}_{3}\right)$ has multiple biological actions, including its antimicrobial effect, which has been beneficial in dentistry, the aqueous presentation being the most used $(20 \mu \mathrm{g} / \mathrm{mL})$, which has similar effects to chlorhexidine. Case report: Male patient of 76 years of age, diagnosed with localized moderate chronic periodontitis, which was treated by aqueous ozone therapy during the initial periodontal phase and surgical phase in debridement flap. Twelve weeks after surgery, the periodontal pockets were eliminated, finding a 3 and $2 \mathrm{~mm}$ groove and good control of dentobacterial plaque. Conclusion: There are no reports about the use of aqueous ozone therapy during a debridement flap. The success of the periodontal treatment consists in the elimination of the causal factor, as well as establishing and maintaining an adequate control of plaque.

Keywords: Ozone, ozone therapy, periodontitis

\section{INTRODUCCIÓN}

L a enfermedad periodontal es una enfermedad que $\checkmark$ afecta los tejidos de soporte del diente, se ha encontrado una prevalencia de $78 \%$ en la población mayor de 60 años, por lo que es considerada la segunda enfermedad bucal más frecuente en México. ${ }^{1}$
Una de las principales causas de la enfermedad periodontal es la acumulación de placa bacteriana, la cual es un acúmulo de bacterias estructuradas, ${ }^{2}$ siendo las bacterias más frecuentes $P$. gingivalis, $T$. forsythia y $T$. denticola. ${ }^{3}$ Este acúmulo estimula la respuesta inflamatoria de los tejidos periodontales, ${ }^{4}$ la cual da como resultado pérdida ósea, bolsas periodontales e inflamación gingival

\footnotetext{
* Universidad Autónoma de Nuevo León, Facultad de Odontología, Postgrado de Periodoncia e Implantología Oral, Monterrey, Nuevo León, México.

Recibido: 29 Agosto 2019. Aceptado para publicación: 19 Agosto 2020.

Citar como: Salazar-Leal JL, Lozano-Belaunzarán RG, Martínez-Sandoval G, Carvajal-Montes de Oca MÁA, Chapa-Arizpe MG. Ozonoterapia como adyuvante en el tratamiento periodontal no quirúrgico y quirúrgico. Revista ADM 2020; 77 (5): 267-271. https://dx.doi.org/10.35366/96147
}

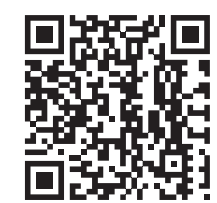




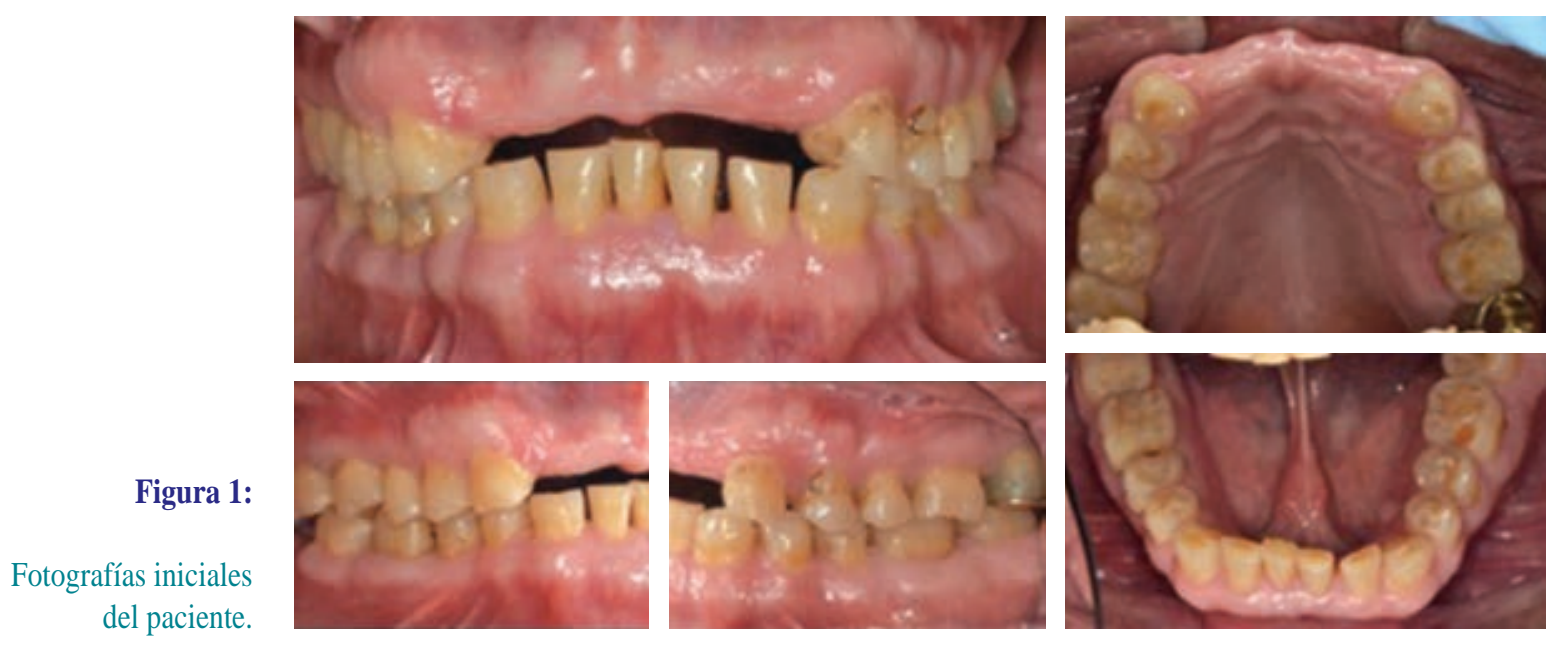

así como sangrado y movilidad dental, lo que genera la pérdida de inserción clínica. ${ }^{5}$

El objetivo de la terapia periodontal consiste en la eliminación de los microorganismos causales, además de la prevención y el control de los factores desencadenantes de la enfermedad. Actualmente se ha demostrado que la periodontitis puede ser tratada con éxito mediante terapia periodontal quirúrgica como no quirúrgica. ${ }^{6}$ Ambas fases del tratamiento buscan la eliminación mecánica de la placa supragingival y subgingival, donde el tratamiento quirúrgico se lleva a cabo mediante de elevación de colgajos para mejorar el acceso y fácil remoción mecánica de bacterias.

Hoy en día se han buscado alternativas adyuvantes del tratamiento para reducir la carga bacteriana presente tales como el ozono, el cual puede ser aplicado en gas, aceite y agua. ${ }^{8} \mathrm{El}$ ozono en odontología ha sido beneficioso debido a su efecto antimicrobiano, ${ }^{9-11}$ siendo la presentación acuosa la más utilizada $(20 \mu \mathrm{g} / \mathrm{mL})$, y su efecto es similar a la clorhexidina. ${ }^{12,13}$

El objetivo del presente estudio es reportar un caso clínico de un paciente de 76 años diagnosticado con periodontitis crónica moderada localizada, quien fue tratado con ozonoterapia acuosa en la fase inicial periodontal y la fase quirúrgica.

\section{PRESENTACIÓN DEL CASO}

Paciente masculino de 76 años de edad, que acudió a consulta al Postgrado de Periodoncia e Implantología Oral
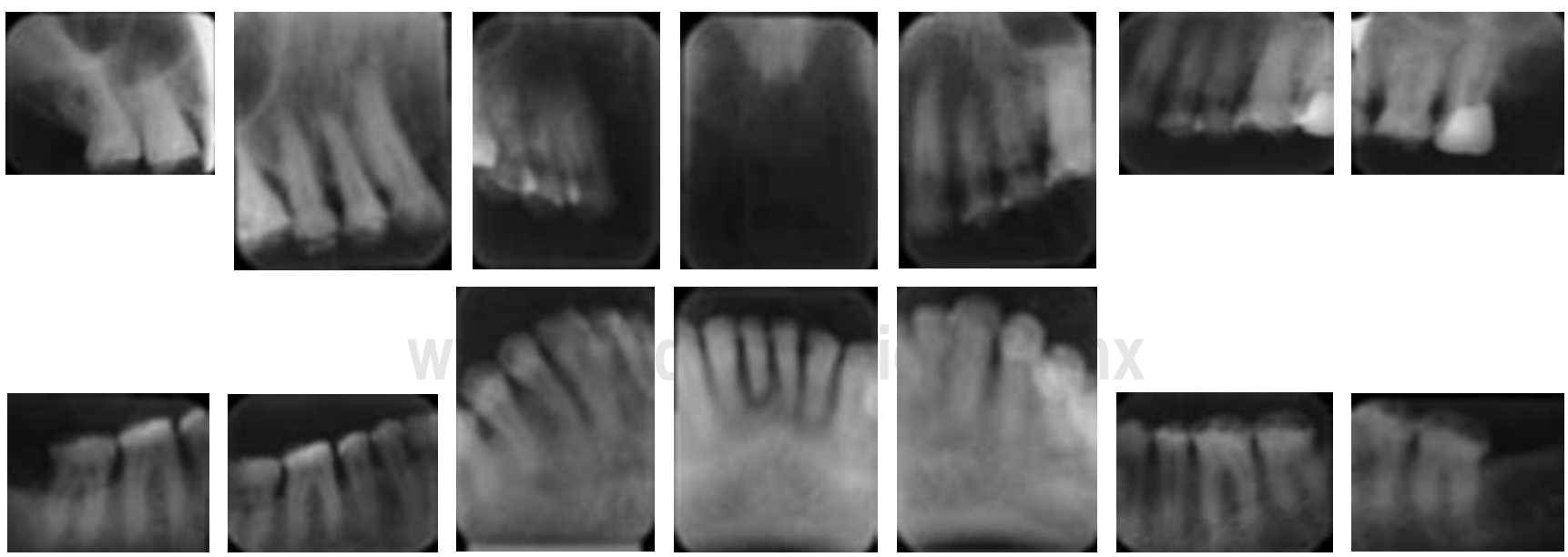

Figura 2: Fotografías iniciales del paciente. 
(FO, UANL) para valoración periodontal. En el interrogatorio de la historia clínica el paciente comenta no tener enfermedades sistémicas ni estar bajo ningún tratamiento médico, no presenta antecedentes de consumo de tabaco o bebidas alcohólicas, por lo que fue clasificado como ASA I. ${ }^{14}$

Dentro de sus antecedentes dentales el paciente fue sometido a extracción de las piezas 1.2, 1.1, 2.1 y 2.2 hace más de cinco años por fractura coronaria, por lo que se colocó una prótesis parcial removible. Además, presenta obturaciones de amalgama y resina en el segmento posterior de ambas arcadas.

El paciente refiere sangrado gingival al cepillado en la zona de molares del cuadrante IV desde hace aproximadamente seis meses. A la exploración física intraoral se encontraron bolsas periodontales de $6 \mathrm{~mm}$ con sangrado al sondeo, placa dentobacteriana y extrusión del sextante anteroinferior, además de presentar contactos prematuros en segmento posterior y atrición generalizada (Figura 1).

En el examen radiográfico se observa una pérdida horizontal moderada en la zona de molares del cuadrante I, III y IV, además de presentar una zona radiolúcida en la pieza 3.1, correspondiendo a una lesión periapical (Figura 2).

Debido a la sintomatología y a los hallazgos clínicos y radiográficos se diagnosticó como periodontitis crónica moderada localizada en la zona de molares del cuadrante III y $\mathrm{VI}$, teniendo un pronóstico general de regular.

Posterior al diagnóstico se realizó la fase higiénica del tratamiento periodontal, la cual consistió en un raspado y alisado radicular de los cuatro cuadrantes bajo la irrigación de agua ozonizada a una concentración de 26.8 $\mu \mathrm{g} / \mathrm{mL}$. Al paciente se le dieron instrucciones de técnica de cepillado de Stillman modificada con un cepillo de cerdas suaves y el uso de hilo dental. Posteriormente se realizó la revaloración periodontal a las cuatro semanas, donde se encontraron bolsas periodontales mayores de $5 \mathrm{~mm}$ en el cuadrante I, por lo que se indicó realizar un colgajo por desbridación.

Previa asepsia y antisepsia y bajo anestesia local, se realizaron incisiones intrasulculares de ángulo línea distal de 1.7 hasta ángulo línea mesial de 1.5, con incisión subadistal (Figura 3A). Se reflejó el colgajo a espesor total, se desbridó y realizó raspado y alisado radicular (Figura 3B) y se suturó con puntos colchoneros verticales externos con seda negra 4-0 (Figura 3C). Se prescribió la ingesta de ketorolaco de $10 \mathrm{mg}$ cada ocho horas por cinco días, además de realizar enjuagatorios de agua ozonizada cada 12 horas por siete días, para posteriormente retirar los puntos de sutura (Figura 3D).

Doce semanas postoperatorias se realizó la revaloración final, donde se encontró un tejido gingival sano, color rosa pálido sin presencia de bolsas periodontales, acúmulo de placa o sangrado al sondeo (Figura 4), por lo que se dio de alta parcial, refiriendo a odontología restauradora e indicando un mantenimiento periodontal cada tres meses.
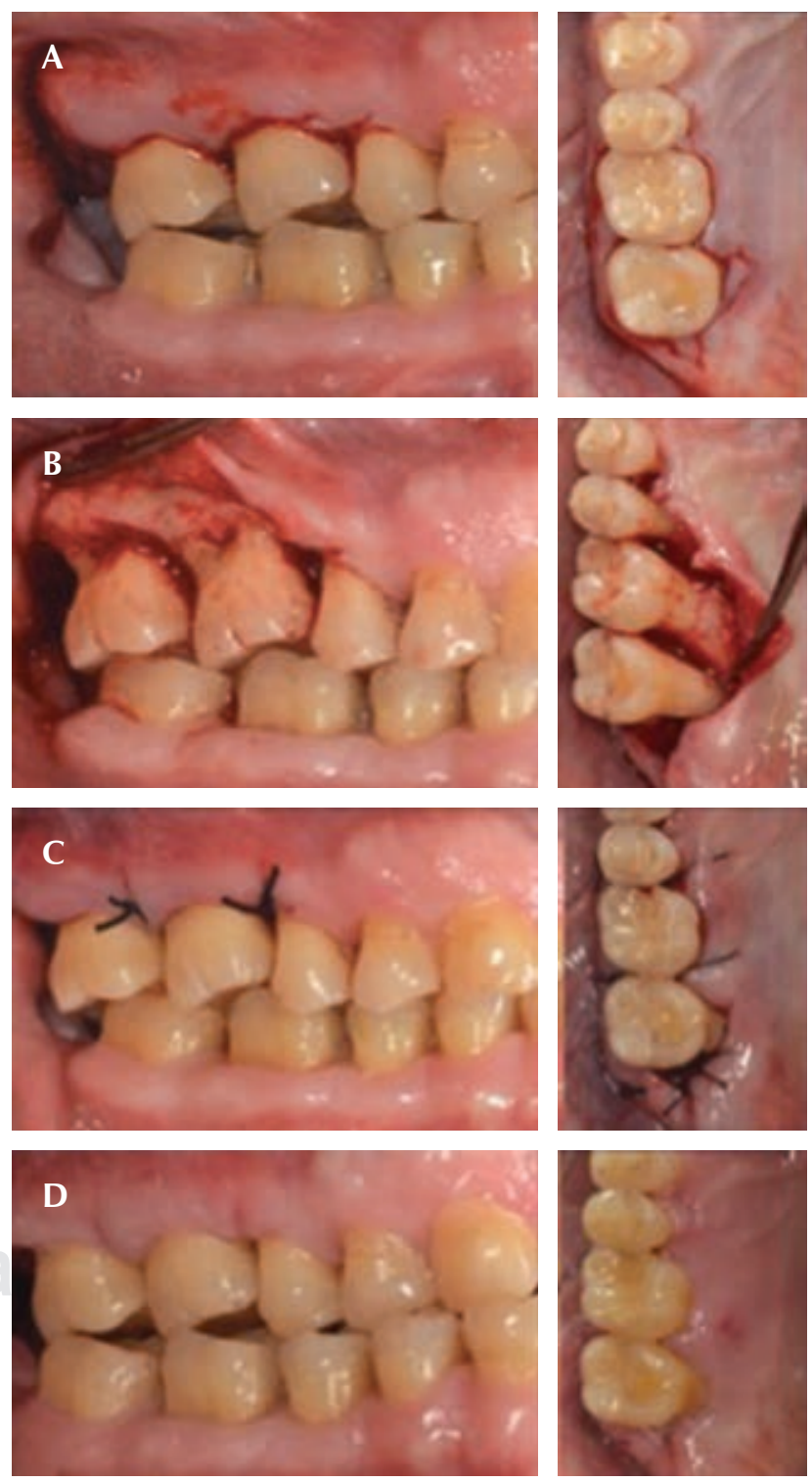

Figura 3: Colgajo por debridación del cuadrante I. 

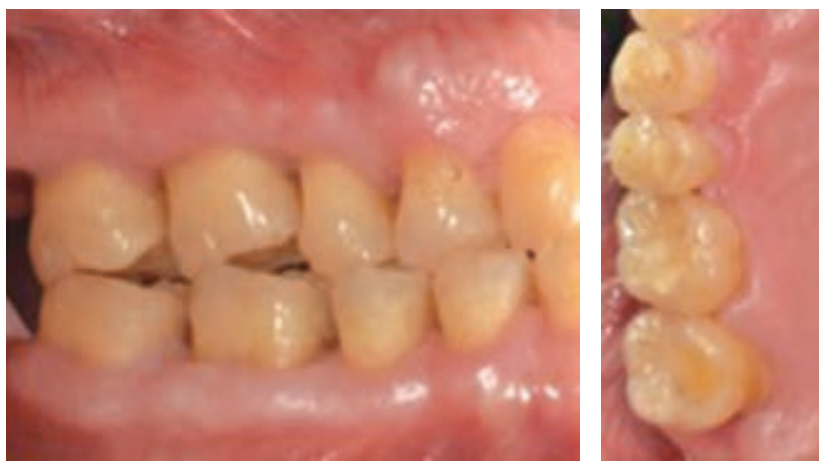

Figura 4: Revisión del sitio tres meses después.

\section{DISCUSIÓN}

Se ha encontrado que el uso de ozonoterapia acuosa durante el tratamiento periodontal no quirúrgico resulta en una disminución de placa dentobacteriana y menor índice de sangrado al sondeo, en comparación con la clorhexidina al $0.2 \%$ que mostró resultados significativamente menores, pudiendo atribuir buenos resultados durante la fase higiénica realizada en el paciente. ${ }^{15}$

En una revisión sistemática se ha demostrado que la irrigación de agua ozonizada en superficies radiculares recién avulsionadas no afecta las células del ligamento periodontal, donde además se encontró una descontaminación de la superficie radicular. ${ }^{16}$

A nuestro conocimiento no se han reportado estudios acerca del uso de agua ozonizada en el tratamiento periodontal quirúrgico; sin embargo, Stübinger y colaboradores han reportado que la irrigación con agua ozonizada durante la osteotomía de terceros molares reduce la posibilidad de infección postoperatoria, además una disminución en el dolor y mejoría en la cicatrización de tejidos blandos. ${ }^{17}$

\section{CONCLUSIÓN}

En este caso clínico, un paciente con periodontitis crónica moderada localizada tratado mediante la irrigación de agua ozonizada durante el tratamiento periodontal quirúrgico y no quirúrgico tuvo una resolución de las bolsas periodontales, presentando un surco gingival sano de $3 \mathrm{~mm}$ a las cuatro semanas posteriores al tratamiento quirúrgico, siendo efectivo el uso de agua ozonizada durante ambas fases del tratamiento periodontal.

\section{AGRADECIMIENTOS}

A CONACYT por la beca otorgada.

\section{BIBLIOGRAFÍA}

1. Fernández R, Olmedo D. Días de estancia Hospitalaria en pacientes con enfermedades respiratorias y enfermedad periodontal. Gac Med Mex. 2017; 153: 21-25.

2. Dentino A, Lee S, Mailhot J, Hefti AF. Principles of periodontology. Periodontol 2000. 2013; 61 (1): 16-53.

3. Costerton JW, Stewart PS, Greenberg EP. Bacterial biofilms: a common cause of persistent infections. Science. 1999; 284 (5418): 1318-1322.

4. Kumar PS, Griffen AL, Barton JA, Paster BJ, Moeschberger ML, Leys EJ. New bacterial species associated with chronic periodontitis. J Dent Res. 2003; 82 (5): 338-844.

5. Plessas A. Nonsurgical periodontal treatment: review of the evidence. Oral Helath Dent Manag. 2014; 13 (1): 71-80.

6. Flemmig TF. Periodontitis. Ann Periodontol. 1999; 4 (1): 32-37.

7. Ojima M, Kanagawa H, Nishida N, Nagata H, Hanioka T, Shizukuishi S. Relationship between attitudes toward oral health at initial office visit and compliance with supportive periodontal treatment. J Clin Periodontol. 2005; 32 (4): 364-368.

8. Morillo LM, Rodriguez JI. Ozonoterapia como adyuvante en el tratamiento periodontal no quirúrgico. Rev Mex Periodontol. 2015; 6 (3): 136-142.

9. Gupta G, Mansi B. Ozone therapy in periodontics. J Med Life. 2012; 5 (1): 59-67.

10. Elvis AM, Ekta JS. Ozone therapy: a clinical review. J Nat Sci Biol Med. 2011; 2 (1): 66-70.

11. Patel PV, Kumar S, Vidya GD, Patel A, Holmes JC, Kumar V. Cytological assessment of healing palatal donor site wounds and grafted gingival wounds after application of ozonated oil: an eighteen-month randomized controlled clinical trial. Acta Cytol. 2012; 56 (3): 277-284.

12. Nogales CG, Ferrari PH, Kantorovich EO, Lage-Marques JL. Ozone therapy in medicine and dentistry. J Contemp Dent Pract. 2008; 9 (4): $75-84$.

13. Hayakumo S, Arakawa S, Mano Y, Izumi Y. Clinical and microbiological effects of ozone nano-bubble water irrigation as an adjunct to mechanical subgingival debridement in periodontitis patients in a randomized controlled trial. Clin Oral Investig. 2013; 17 (2): 379-388.

14. Maloney WJ, Weinberg MA. Implementation of the American Society of Anesthesiologists physical status classification system in periodontal practice. J Periodontol. 2008; 79 (7): 11241126.

15. Kshitish D, Laxman VK. The use of ozonated water and $0.2 \%$ chlorhexidine in the treatment of periodontitis patients: a clinical and microbiologic study. Indian J Dent Res. 2010; 21 (3): 341 348.

16. Azarpazhooh A, Limeback $\mathrm{H}$. The application of ozone in dentistry: a systematic review of literature. J Dent. 2008; 36 (2): 104-116.

17. Stübinger S, Sader R, Filippi A. The use of ozone in dentistry and maxilofacial surgery: a review. Quintessence Int. 2006; 37 (5): 353-359. 
Correspondencia:

Dr. Jorge Luis Salazar-Leal

Postgrado de Periodoncia e Implantología Oral,

Facultad de Odontología,

Universidad Autónoma de Nuevo León.

E Aguirre Pequeño y Silao,
Mitras Centro, 64460

Monterrey, N.L. México,

Tel: 528183294000 ,

ext. 3192 y 3100 .

E-mail: drjorgesalazar93@gmail.com

investigacionperiouanl@gmail.com 